\title{
Potential impact of climate change on the be
}

\author{
Castellanos-Potenciano, Blanca Patricia; Gallardo-López, Felipe; Sol- \\ Sánchez, Ángel; Landeros-Sánchez, Cesáreo; Díaz-Padilla, Gabriel; \\ Sierra-Figueredo, Pablo; Santibáñez-Galarza, José Luis; Editor \\ académico Dr. Prof. Carlos Zúniga-González
}

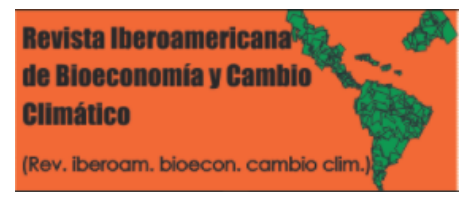

\author{
iD Blanca Patricia Castellanos-Potenciano \\ bcastellanos@colpos.mx \\ Colegio de Postgraduados, Campus Veracruz., México \\ (iD Felipe Gallardo-López \\ felipegl@colpos.mx \\ Colegio de Postgraduados, Campus Veracruz., México \\ (iD) Ángel Sol-Sánchez \\ sol@colpos.mx \\ Colegio de Postgraduados, Campus Tabasco., México \\ (iD) Cesáreo Landeros-Sánchez \\ clandero@colpos.mx \\ Colegio de Postgraduados, Campus Veracruz., México \\ Gabriel Díaz-Padilla \\ diaz.gabriel@inifap.gob.mx \\ 3Instituto Nacional de Investigaciones Forestales, \\ Agrícolas y Pecuarias,, México \\ (iD) Pablo Sierra-Figueredo \\ sierrafp@gmail.com \\ Instituto de Geofísica y Astronomía, Agencia de Medio \\ Ambiente, Cuba \\ José Luis Santibáñez-Galarza \\ jlsantivañez44@gmail.com \\ CATIE-Bolivia, Bolivia \\ Editor académico Dr. Prof. Carlos Zúniga-González \\ Universidad Nacional Autónoma de Nicaragua, León, \\ Nicaragua
}

Revista Iberoamericana de Bioeconomía y Cambio Climático

Universidad Nacional Autónoma de Nicaragua, León, Nicaragua ISSN-e: 2410-7980

Periodicidad: Semestral

vol. 2, núm. 1, 2016

czuniga@ct.unanleon.edu.ni

Recepción: 13 Mayo 2015

Aprobación: 10 Enero 2016

URL: http://portal.amelica.org/ameli/journal/394/3941750035/

DOI: https://doi.org/10.5377/ribcc.v2i1.5673
Resumen: El cambio climático representa el mayor reto para la humanidad en el siglo XXI, proyectando posibles cambios sociales, económicos y ecológicos a nivel global; lo que hace necesario plantear estrategias de adaptación y mitigación en las actividades del sector primario que permitan reducir el riesgo a esos cambios. Por lo que el objetivo de esta revisión fue analizar las investigaciones realizadas sobre los efectos del cambio climático en la apicultura a partir de dos de sus elementos básicos: las relaciones fisico-biologicos y los riesgos socioeconómicos. Los impactos potenciales se clasificaron en directos considerando la respuesta intra e inter-específica de la flora melífera y las abejas; e indirectos, enfocados a las afectaciones socioeconómicas. Se encontró que las afectaciones de orden directo conllevan a respuestas intra-específicas de las especies de plantas como la movilidad espacio temporal hacia latitudes más elevadas y a la dinámica poblacional de las colonias de abejas. Los cambios indirectos incluyen un sentido económico y social por el riesgo de la rentabilidad a consecuencia del incremento en las prácticas de adaptación, desembocando por consiguiente en un posible abandono de la actividad. Concluyendo en la evidente necesidad de trabajos regionales integrales e interdisciplinarios que contribuyan a prever la respuesta biológica de las especies involucradas en la actividad (desde el paradigma cuantitativo) y la comprensión del fenómeno (desde el paradigma cualitativo), resultado de la incertidumbre de los apicultores, quienes bajo su propio esquema de cogniciones deciden invertir o no, en prácticas de manejo que ayuden a mantener la producción.

Palabras clave: Abejas, Impactos cambio climático, Riesgo apícola.

Abstract: Climate change is the greatest challenge for humanity in the twenty-first century, projecting potential social, economic and ecological conditions globally; It is making it necessary to consider adaptation and mitigation strategies in the primary sector activities to reduce the risk to those changes. So the aim of this review was to analyze the research on the effects of climate change on beekeeping from two of its basic elements: the physical and biological relationships and socio-economic risks. Potential impacts were classified into direct consideration intra- and inter-specific response of flora and honey bees; and indirect, focused on the socio-economic damages. It was found that the effects of direct order lead to intra-specific plant species such as temporary space mobility towards higher 
latitudes and population dynamics of bee colonies answers. Indirect changes include an economic and social sense for risk profitability as a result of the increase in adaptation practices, leading consequently to a possible abandonment of the activity. Concluding the obvious need for comprehensive regional and interdisciplinary work that contribute to provide the biological response of the species involved in the activity (from the quantitative paradigm) and understanding of the phenomenon (from the qualitative paradigm), due to the uncertainty of beekeepers who, under his own scheme of cognitions they decide to invest or not in management practices that help maintain production.

Keywords: Bees, climate change impacts, Beekeeping risk.

\section{INTRODUCCIÓN}

El cambio climático representa el mayor reto para la humanidad en el siglo XXI, el calentamiento global implícito ha incrementado en $1^{\circ} \mathrm{C}$ la temperatura global con respecto al inicio de la era industrial (Conde, 2010), por las emisiones de gases efecto invernadero (GEI), que provocan efectos negativos ambientales, económicos y sociales (Botello and Villanueva-Fragoso, 2011).

En este contexto el Panel Intergubernamental sobre el Cambio Climático (IPCC) publicó la proyección de escenarios climáticos alternativos (RCPs) basados en las emisiones de GEI y en los patrones y tendencias de la temperatura en la superficie a escala continental de continuar las emisiones de esos gases, proyectando los posibles cambios sociales, económicos y ecológicos a nivel global, involucrando los posibles efectos sobre las actividades agropecuarias del sector primario y planteando alunas estrategias de adaptación y mitigación que permitan reducir el riesgo y la vulnerabilidad a esos cambios (IPCC, 2014a; IPCC, 2014b).

La apicultura como parte de este sector, es la práctica dedicada a la cría y explotación racional de las abejas, que al igual que cualquier otra actividad agropecuaria de temporal, depende de un intervalo de condiciones climáticas estables para su óptimo desarrollo (Delgado et al., 2012).

Además de brindar el servicio ambiental de polinización (del cual dependen 286 especies cultivadas que se destinan para la alimentación y producción de materias primas) (Coro, 2009); la producción de miel a nivel mundial es el principal producto que se comercializa en el mercado, con incrementos en el total del volumen de producción internacional en 135 países (FAO, 2015).

México es el país que ocupa el octavo lugar en producción de miel después China, Turquía, Argentina, Ucrania, USA, Federación de Rusia e India y la quinta posición como exportador de este producto; generando ingresos por más de 90 millones de dólares (AUSAID, 2013; FAO, 2015, SIAP, 2015). Por lo tanto la prevalencia de las condiciones ambientales óptimas para el desarrollo de la abeja melífera es fundamental para el desempeño de la actividad apícola.

Los cambios en el clima contemplados en los escenarios del IPCC, podrían impactar de forma potencial en la apicultura al incrementar en sentido negativo el riesgo de la actividad con base en dos sentidos: directo, considerando la respuesta intra e inter específica de la flora melífera y las abejas, e indirecto, enfocado a las afectaciones socioeconómicas de los apicultores por los riesgos de producción y la incertidumbre que conlleva (Figura 1).

\section{NotAS DE AUTOR}




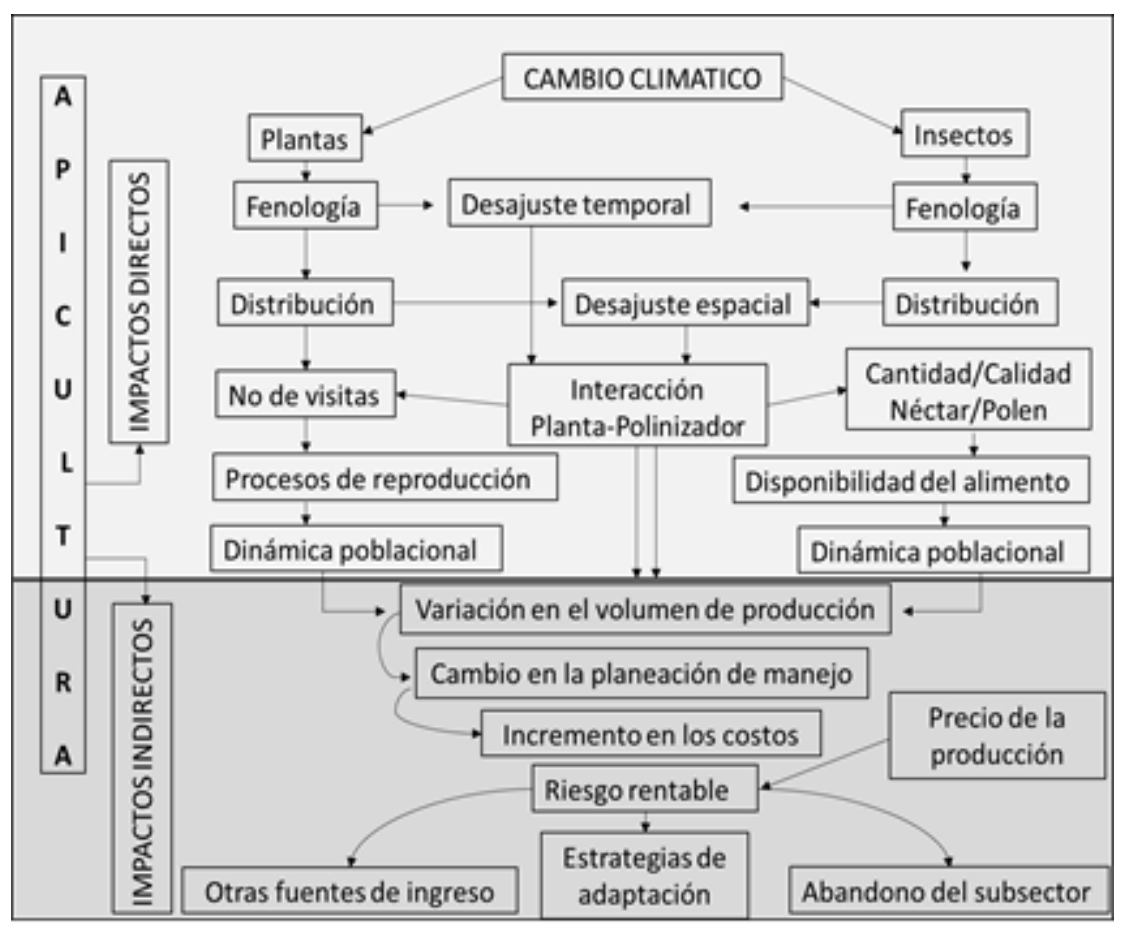

FIGURA1

Esquema del impacto del cambio climático en la apicultura. Modificado de Hegland et. al.,(2009).

Con base a lo expuesto, esta revisión analiza los efectos del cambio climático en la apicultura a partir de dos de sus elementos básicos, las relaciones fisico-biologicos, considerando a las plantas como fuente de alimento y las abejas como organismos transformadores, a partir de la respuesta fenológica de las especies y sus implicaciones en la relación planta-insecto frente a las variaciones de los factores meteorológicos de temperatura, humedad y precipitación. Así como los riesgos socioeconómicos que podrían representar para la apicultura, a pesar de los incrementos en la producción de miel a nivel mundial reportados por la FAO.

\section{Impactos Directos}

1.1 Influencia del Cambio Climático en la vegetación. Ante la inquietud del impacto del cambio climático sobre la comunidad vegetal se han realizado investigaciones que documentan la tendencia de diferentes especies botánicas ante tales anomalías. Así por ejemplo se sabe que las plantas han respondido a las variaciones climáticas principalmente desde el eje de la adaptación y migración (Alvarado et al., 2002;Bellard et al., 2012).

Estos cambios han sido evaluados en los últimos años a través de los modelos de predicción climática que incluyen múltiples variables bajo los diferentes escenarios de cambio climático (Jorquera and Orrego, 2010). Alvarado et al., (2002), reporta que el principal impacto sobre la vegetación se refleja en la fenología de desarrollo de la misma, principalmente por la variación en la temperatura y la acumulación de CO2. Por su parte Gordo and Sanz (2005) evaluaron la respuesta de las fases fenológicos de 45 especies vegetales (25 cultivadas y 20 nativas) con una base de datos climáticos de temperatura y precipitación de1943 al 2003, reportando que los incrementos en la temperatura fueron el principal factor que impacto en el desarrollo de las floraciones de primavera principalmente en el mes de marzo.

La tendencia al incremento de las temperaturas globales hace necesario evaluar su impacto en las especies cultivadas. Jorquera and Orrego (2010), calcularon los cambios fenológicos de Vitis vinífera L. a través de modelos denominados AOGCM (Atmospheric and Ocean Global Climatic Model) sugiriendo en términos 
generales un adelanto de las fases fenológicas en el escenario menos cálido (B2) del IPCC (2007), así como la falta de capacidad de los espacios actuales para el desarrollo del cultivo.

La movilidad de los nutrientes en el suelo (además de los factores climáticos dominantes) contribuyen con la movilidad espacial de las especies; los procesos de erosión, lixiviación, sequía y lluvias torrenciales incrementa el riesgo de pérdida de nutrientes, la disposición de los mismos (Sardans and Peñuelas, 2012; Granados and Sarabia, 2013) y la concentración y volumen de producción de néctar (Hidalgo and Cabezudo, 1995).

Aunque los estudios de la producción del néctar de las flores se ha relacionado con el tipo de polinizador y síndrome floral, se han documentado diferencias en la producción de néctar entre regiones geográficas, entre poblaciones, plantas de una misma población y entre las flores de una misma planta (Herrera, 1995; Devoto et al., 2006). Además de variaciones por época del año, meses y horas del día, por la influencia de factores abióticos como la temperatura y humedad (Paiaro et al., 2012).

Lo anterior demuestra el efecto que las variaciones abióticas tienen sobre la vegetación en diferentes niveles desde los biogeográficos hasta los fisiológicos y de acuerdo al tipo de modelos de proyección, escenario o técnica de comprobación, la respuesta de los cambios en las plantas puede proyectarse de leves a moderados, restringiendo la disponibilidad de alimentos para las abejas.

1.2 Influencia del cambio climático en las abejas. En general los insectos responden de manera rápida a los cambios de temperatura y precipitación en el ambiente. (Hegland et al., 2009; Ladány and Horváth, 2010; Hódar et al., 2012). Por ser organismos ectodérmicos y con ciclos de vida cortos, están limitados en su distribución por las bajas temperaturas registradas en las latitudes más altas (Wilson and Maclean, 2011). El incremento en la temperatura que trae consigo el cambio climático proyecta un cambio en la distribución de estas especies hacia latitudes más elevadas, permitiendo a las poblaciones de insectos ampliar y movilizar su hábitat (Regniere, 2009).

Musolini y Saulich (2012) afirman que los insectos responden al cambio climático en diferentes sentidos de acuerdo a las características propias de cada especie (Figura 2), proponiendo seis categorías que involucran cambios intra-especificos y sus interacciones ecológicas.

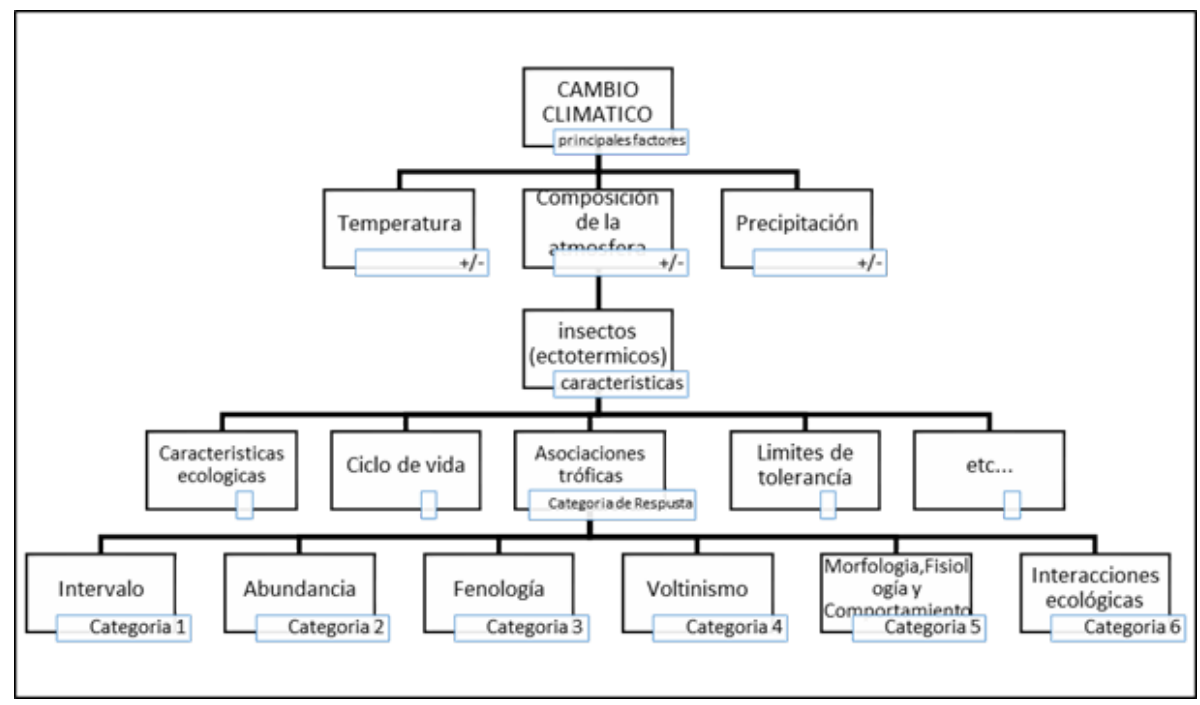

FIGURA 2.

Respuesta de las especies de insectos ante las variaciones de los principales factores del cambio climático. Elaboración propia basada en Musolini y Saulich (2012).

Como muestran las Figuras 1 y 2 la temperatura interviene directamente sobre el ciclo de vida de los insectos (Musolin and Saulich, 2012; Kiritani, 2013) ya sea reduciendo la mortalidad de la población en 
invierno o alargando la temporada de reproducción como respuesta de cada especie ante las variaciones del Cambio Climático (Arribas et al., 2012).

Estos cambios fenológicos causados por las variaciones térmicas, pueden interrumpir el ajuste temporal de la interacción planta-insecto (Redi et al., 2012; Aluja et al., 2014). Como por ejemplo los Hemipteros del genero Nezara spp. que presenta variaciones en su ciclos de reproducción por stress térmico ( $N$. viridula de regiones tropicales y $N$. antennata de las regiones de Asia) y especies del orden Coleoptera, Hortoptera, Hemiptera e Hymenoptera, en especial las abejas, que ponen en evidencia la susceptibilidad de las poblaciones de insectos al calentamiento global como efecto del cambio climático (Kiritani, 2013).

Apis mellifera L. es una especie de abeja que requiere de condiciones de temperatura $\left(34-35^{\circ} \mathrm{C}\right)$ dentro de la colmena para su óptimo desarrollo (Le Conte y Navajas, 2008), por lo que la temperatura del ambiente representan el factor abiótico más importante que influye directamente en el óptimo desarrollo de la cría y por consiguiente en el comportamiento y actividades de la población (Mendizabal, 2005; Ali, 2011) (Figura 3).

Tenczar et al. (2014) afirma que A. mellifera es capaz de adaptar su nivel de actividad de pecoreo por los estímulos externos (disposición de alimento) y a estímulos internos (demografía de la colonia) que se presenten.

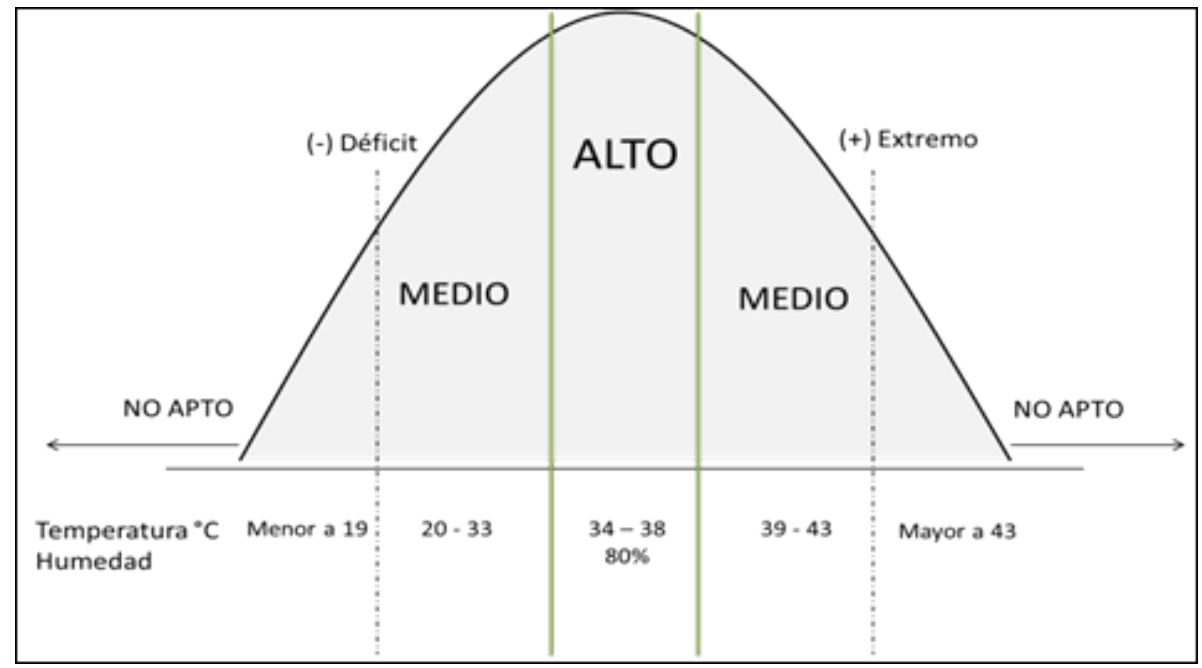

FIGURA 3.

Requerimientos de temperatura y humedad ideal para estimular la actividad de pecoreo en la abeja de miel Apis mellifera L.

Elaboracion propia

Con base al incremento en la temperatura global de 1.8 a $4{ }^{\circ} \mathrm{C}$ y cambios en los patrones de lluvia de los escenarios de proyección (IPCC, 2007; Conde, 2010), se espera que las abejas respondan limitando la postura de huevos y disminuyan la actividad en el pecoreo de néctar y polen, por agua. Está demostrado que la especie A. florae disminuye su actividad de pecoreo en cultivos de mango, con temperaturas superiores a $32^{\circ} \mathrm{C}$ (Redi et al., 2012) y A. mellifera ha presenta un comportamiento similar en un cultivo de Guzotia abyssinica (Asteraceae), bajo las mismas condiciones de temperatura que A. florae (Gebremedhn et al., 2014); dejando en evidencia la vulnerabilidad del género y la especie ante los incrementos de temperatura principalmente, sin embargo la presencia de otro factores climáticos interfiere en las actividades de una colmena.

La presencia de precipitación limita las actividades de pecoreo limitando el tiempo de vuelo, lo que implica utilizar el alimento acumulado en las colmenas (Delgado, 1984) así como la pérdida de néctar y el polen en las plantas, reduciendo la cantidad y calidad del alimento disponible (Mendizabal, 2005). Por lo tanto los cambios en los patrones de lluvias citados por el IPCC, podría reducir en cantidad y calidad la disponibilidad de alimento en los meses que debiera ser abundante o, prolongar las estaciones de lluvia y reducir la actividad de pecoreo. (vanEngelsdorp and Meixner, 2010). 
Con respecto a la presencia de humedad relativa y la dinámica de pecoreo las abejas, se correlacionan de forma negativa (Abrol, 2010) y aunque no influye en algún estadío en particular, interviene en la capacidad de pérdida y adquisición de calor en el interior de la colmena (Delgado, 1984), involucrando flujos de energía en el transporte del néctar y el polen, además de un incremento en el gasto energético para la eliminación de humedad del alimento almacenado (Kovac and Stabentheiner, 2011; Gebremedhn et al., 2014).

\section{Impactos Indirectos}

El desequilibrio entre la fenología de las plantas y la dinámica de las abejas (Ma and Ma, 2012), producto de las variaciones climáticas, ocasiona que el inicio prematuro de las floraciones se desfase con la fenología de las colmenas, ocasionando que el acopio de miel pueda ser deficiente (Le Conte and Navajas, 2008; Ladány and Horváth, 2010; Pico, 2011) y como consecuencia reducir el volumen de cosecha de miel para el apicultor (Redi et al., 2012) quien debe de modificar sus labores de manejo para mantener la producción y la salud de las colmenas (Guzmán and Correa, 2012).

La escases de alimento, el desajuste en la dinámica poblacional y las limitaciones de pecoreo no son los únicos impactos potenciales a consecuencia del cambio climático, los incrementos de temperatura, la presencia prolongada de lluvia y humedad, incrementan la vulnerabilidad hacia la presencia de enfermedades y plagas propias de las abejas, como Varroa destructor, ectoparásito considerado la principal plaga que amenaza las colmenas (Medina-Flores et al., 2011; Giacobino et al., 2014) y que debe sus niveles de infestación a la dinámica reproductiva de la propia abeja y las condiciones climáticas prevalecientes (Frey et al., 2013), debilitando las colmenas por desnutrición animal y favoreciendo el desarrollo de otros patógenos (Guzmán and Correa, 2012) que limitan la producción de miel (Huerta, 2008).

Por lo que los impactos potenciales del cambio climático en la producción de miel son un problema complejo que involucra, la falta de condiciones climáticas que impulsen altos rendimientos y mejoren la productividad (CREEBBA, 2005). De modo que los apicultores están sujetos a un riesgo económico que deriva en que vulnera la rentabilidad de los apíarios (Al-Ghamdi et al., 2014) donde la aplicación de medicamentos, la alimentación artificial y la movilización de colmenas principalmente, como estrategias de adaptación para la producción, representan costos variables en continuo incremento y que deben ser recuperados por la venta de la miel en el mercado, restringiendo la capacidad de generar utilidades que mantengan la rentabilidad de la actividad apícola (Magaña and Leyva, 2011).

Además es necesario integrar como impacto indirecto la suma en la posible asociación entre la variabilidad de la Actividad Solar, Geomagnética, el clima terrestre y la productividad apícola (Pérez y Sierra, 1993, Sierra y Baca, 2014). Que muestran cómo la variabilidad de dichos agentes del clima espacial modula, de cierta manera, la productividad apícola a corto y largo plazo, lo que pudiera tomarse en cuenta para el planteamiento de modelos más integrales y una mejor planificación del esfuerzo productivo.

Por lo tanto el impacto socioeconómico negativo en la apicultura inicia con el desequilibrio del clima y las plantas, (Musimba et al., 2001) que obliga a implementar estrategias de producción que incrementan el costo de inversión (Delgado et al., 2012) afectando el nivel de rentabilidad que por sí mismo ya es variable, por lo que se requiere mantener o mejorar los niveles de producción que permitan la recuperación económica de los costos de inversión.

A nivel mundial se reportan malas prácticas de manejo para mantener la producción de miel, como la sobre-alimentación de las abejas con azúcar y otros tipos de sacarosa durante los periodos de abundante néctar (Yilmaz et al., 2014). o bien la adición de fructosa o glucosa industrial que da lugar a un cambio de la relación fructosa/glucosa (1-1.2 en miel natural) en la miel adulterando sus propiedades e incrementando el volumen para comercializar (Puscas et al., 2013).

La hipótesis sobre la comercialización de las mieles adulteradas, aun mantiene un respaldo escaso en revistas arbitradas, los medios de comunicación popular a nivel mundial han sido los encargados de mantener la alerta 
de esta problemática, exponiendo, que debido a la escases de la producción, del 30 al 50\% de la miel en el mercado mundial puede ser adulterada y podría explicar los incrementos de producción global, a pesar de la problemática del cambio climático y sus impactos potenciales en la apicultura actual.

\section{CONCLUSIONES}

Las evidencias de los potenciales impactos del cambio climático son cada vez más concretas, las investigaciones recientes muestran el riesgo en la interacción biológica entre las especies de plantas e insectos asociados a ellas.

La respuesta intra-específicas de las plantas, como la movilidad espacio temporal hacia latitudes más elevadas y a los cambios, en la dinámica poblacional de las colonias de abejas que registran reducción en su tamaño poblacional a causa de la poca actividad de pecoreo y a la escases de alimento, involucran impactos potenciales de orden directo, que se reflejan en el eje socio-económico, al exponer el riesgo del ingreso económico del apicultor por los incrementos en los costos de producción que responden a impactos potenciales indirectos.

Aunque los efectos potenciales del cambio climático sobre la apicultura aún son generales y limitados, se hace evidente la necesidad de realizar trabajos de investigación integrales e interdiciplinarios que evalúen y respalden la problemática planteada (desde un paradigma cuantitativo y cualitativo), así como los impactos potenciales de las variaciones del cambio climático por regiones, con base a escenarios de proyección climática, además de la inclusión de variables del Clima Espacial y su posible impacto tanto en la flora melífera como en la población apícola que permitirían analizar un escenario más integral con vistas a la mitigación de la variabilidad climática y prevención del comportamiento productivo.

En aras de plantear estrategias de adaptación ante los impactos potenciales del cambio climatico y la incertidumbre en la rentabilidad del sistema de producción para los apicultores, quienes bajo su propio esquema de cogniciones deciden invertir en prácticas de adaptación que ayuden a mantener la producción o, al abandono de la apicultura.

Los autores agradecen a la línea prioritaria de investigación 8 del Colegio de Postgraduados " Impacto y Mitigación del Cambio climático, por el apoyo y facilidades para el desarrollo de la Presente Investigación.

\section{LITERATURA CITADA}

Abrol D. P.(2010). Foraging behaviour of apis florea f., an important pollinator of allium cepa l. Journal of Apicultural Research 49, (4): 318-325.

Al-Ghamdi A.; Adgaba N.; Getachew A. and Tadesse Y.(2014). New approach for determination of an optimum honeybee colonies carrying capacity based on productivity and nectar secretion potential of bee forages species. Saudi Journal of Biological Sciences (0):

Ali M. A. M.(2011). Comparative study for evaluating two honey bee races, apis mellifera jementica (indigenous race) and apis mellifera carnica (carniolan race) in brood production, population development and foraging activity under the environmental conditions of the central region of the kingdom of saudi arabia. Annals of Agricultural Sciences 56, (2): 127-134.

Aluja M.; Birke A.; Ceymann M.; Guillén L.; Arrigoni E.; Baumgartner D.; Pascacio C. and Samietz J.(2014). Agroecosystem resilience to an invasive insect species that could expand its geographical range in response to global climate change. Agriculture, Ecosystems \& Environment 186, (0): 54-63.

Alvarado M. A.; Foroughbakhch P. R.; Jurado Y. E. y Rocha A.(2002). El cambio climático y la fenología de las plantas. Ciencia UANL 5, (4): 493-500.

Arribas P.; Abellán P.; Velasco J.; Bilton D. T.; Lobo J. M.; Millán A. y Sánchez-Fernández D. (2012). La vulnerabilidad de las especies frente al cambio climático, un reto urgente para la conservación de la biodiversidad. 
AUSAID. (2013). Trade information brief (honey). Trade and Industrial Policy Estrategies (TIPS). Australian Agency for International Development Australian. p. 42.

Bellard C.; Bertelsmeier C.; Leadley P.; Thuiller W. and Courchamp F.(2012). Impacts of climate change on the future of biodiversity. Ecology Letters 15, (4): 365-377.

Botello A. V. and Villanueva-Fragoso S. (2011). Introducción 1-14p. In: V B. A.; S V.-F.; J G. y Rojas G. J. L.s (eds.). Vulnerabilidad de las zonas costeras mexicanas ante el cambio climático (segunda edición). Universidad Autónoma Metropolitana-Iztapalapa, unam-icmyl, Universidad Autónoma de Campeche. México. p. 754.

Conde A. A. (2010). El cambio climático. De lo inequivoco a lo incierto 17-34p. In: Delgado G. C.; Gay C.; Imas M. y Martinez A.s (eds.). México frente al cambio climático. Retos y oportunidades. México : UNAM, Centro de Ciencias de la Atmósfera : Centro de Investigaciones Interdisciplinarias en Ciencias y Humanidades : Programa de Investigación en Cambio Climático : Pro-grama Universitario de Medio Ambiente, 2010. México. p. 240.

Coro A. M. (2009). La crisis de los polinizadores. Biodiversitas. CONABIO. pp. 1-5.

Delgado D. I.; Eglee P. M.; Galindo-Cardona A.; Giray T. and C. R.(2012). Forecasting the influence of climate change on agroecosystem services: Potential impacts on honey yields in a small-island developing state. Psyche 2012: $1-10$.

Delgado R. M. A. (1984). Apicultura y dinámica de poblaciones de apis mellifera en una zona trópical húmeda. Facultad de Ciencias. Universidad Nacional Autónoma de México. México, D.F. p. 90.

Devoto M.; Montaldo N. H. and Medan D.(2006). Mixed hummingbird: Long-proboscid-fly pollination in 'ornithophilous'embothrium coccineum (proteaceae) along a rainfall gradient in patagonia, argentina. Austral Ecology 31, (4): 512-519.

FAO. (2015). Producción/ganaderia primaria. FAOSTAT.

Frey E.; Odemer R.; Blum T. and Rosenkranz P.(2013). Activation and interruption of the reproduction of varroa destructor is triggered by host signals (apis mellifera). Journal of Invertebrate Pathology 113, (1): 56-62.

Gebremedhn H.; Tadesse A. and Belay T.(2014). Relating climatic factors to foraging behavior of honeybees (apis mellifera) during blooming period of guizotia abyssinica (l.F). Livestock Research for Rural Development 26, (4):

Giacobino A.; Cagnolo N. B.; Merke J.; Orellano E.; Bertozzi E.; Masciangelo G.; Pietronave H.; Salto C. and Signorini M.(2014). Risk factors associated with the presence of varroa destructor in honey bee colonies from east-central argentina. Preventive Veterinary Medicine 115, (3-4): 280-287.

Gordo O. and Sanz J.(2005). Phenology and climate change: A long-term study in a mediterranean locality. Oecologia 146, (3): 484-495.

Granados R. R. and Sarabia R. A.(2013). Cambio climático y efectos en la fenología del maíz en el ddr-toluca.

Guzmán N. E. y Correa B. A. (2012). Patología, diagnostico y control de las principales enfermedades y plagas de las abejas meliferas. In: SAGARPAs (ed.). p. 157p.

Hegland S. J.; Nielsen A.; Lázaro A.; Bjerknes A.-L. and Totland Ø.(2009). How does climate warming affect plantpollinator interactions? Ecology Letters 12, (2): 184-195.

Herrera C. M.(1995). Microclimate and individual variation in pollinators: Flowering plants are more than their flowers. Ecology 76, (5): 1516-1524.

Hidalgo M. y Cabezudo B.(1995). Producción de néctar en matorrales del sur de españa (andalucía). Acta Botanica Malacitana 20: 123-132.

Hódar J. A.; Zamora R. and Cayuela L. (2012). Cambio climático y plagas: Algo más que el clima.

Huerta G. (2008). La apicultura en el desarrollo. Presencia. INTA EEA Bariloche, Argentina. pp. 25-27.

IPCC. (2014a). Cambio climático 2014 impactos adaptación y vulnerabilidad. In: II G. d. t.s (ed.). p. 30.

IPCC. (2014b). Cambio climático 2014 mitigación del cambio climático. In: Grupo de trabajo IIIs (ed.). Quinto Informe de Evaluación del Grupo Intergubernamental de Expertos sobre el Cambio Climático,. p. 35.

Jorquera F. E. and Orrego V. R.(2010). Impacto del calentamiento global en la fenología de una variedad de vid cultivada en el sur de chile. Agrociencia 44: 427-435. 
Kiritani K.(2013). Different effects of climate change on the population dynamics of insects. Applied Entomology and Zoology 48, (2): 97-104.

Kovac H. and Stabentheiner A.(2011). Thermoregulation of foraging honeybees on flowering plants: Seasonal variability and influence of radiative heat gain. Ecological Entomology 36, (6): 686-699.

Ladány M. and Horváth L.(2010). A review of the potential climate change impact on insect populations - general and agricultural aspects. Applied Ecology and Enviromental Research 8, (2): 143-152.

Le Conte Y. and Navajas M.(2008). Climate change: Impact on honey bee populations and diseases. Rev. sci. tech. Off. int. Epiz. 27, (2): 499-510.

Ma G. and Ma C.-S.(2012). Effect of acclimation on heat-escape temperatures of two aphid species: Implications for estimating behavioral response of insects to climate warming. Journal of Insect Physiology 58, (3): 303-309.

Magaña M., M. y Leyva M., E.(2011). Costos y rentabilidad del proceso de producción apícola en méxico. Contaduria y Administracion 235: 99-119.

Medina-Flores C. A.; Guzmán-Novoa E.; Aréchiga-Flores C. F.; Aguilera-Soto J. I.y Gutiérrez-Piña F. J.(2011).Efecto del nivel de infestación de varroa destructor sobre la producción de miel de colonias de apis mellifera en el altiplano semiárido de méxico. Revista mexicana de ciencias pecuarias 2:313-317.

Mendizabal F. (2005). Abejas. Buenos Aires-Republica de Argentina. 255 pp.

Musimba N. K.; Nyariki D. M. and Mutungi E. M.(2001). The socio-economics, culture and ecology of beekeping among the akamba community of sourthern kenya. J. Hum. Ecol 12, (3): 207-216.

Musolin D. L. and Saulich A. K.(2012). Responses of insects to the current climate changes: From physiology and behavior to range shifts. Entomological Review 92, (7): 715-740.

Paiaro V.; Oliva G. E.; Cocucci A. A. y Sérsic A. N.(2012). Caracterización y variación espacio-temporal del néctar en anarthrophyllum desideratum (fabaceae): Influencia del clima y los polinizadores. Boletín de la Sociedad Argentina de Botánica 47: 375-387.

Pérez A., Sierra P., (1993). Preliminary Resultats About Possible Relation Between Heliomagnetical Disturbances and Honey Producction in Cuba. APIMONDIA. 33rd International Apicultural Congress, Beijing, China, Set. / 1993.

Pico A. M. (2011). Impacto del cambio climático en la apicultura. Apicultura sin fronteras. Periodico de distribucion mundial. Rodrigo Gonzales. pp. 4-8.

Puscas A.; Hosu A. and Cimpoiu C.(2013). Application of a newly developed and validated high-performance thinlayer chromatographic method to control honey adulteration. Journal of Chromatography A 1272: 132-135.

Redi R.; Verghese A. and Rajan V.(2012). Potential impact of climate change on honeybees (apis spp.) and their pollination services. Pest Management in Horticultural Ecosystems 18, (2): 121-127.

Regniere J.(2009). Prediccion de la distribucion continental de insectos a partir de la fisiologia de las especies. Unasylva 60, $(231 / 232): 37-42$.

Sardans J. and Peñuelas J.(2012). The role of plants in the effects of global change on nutrient availability and stoichiometry in the plant-soil system. Plant Physiology 160, (4): 1741-1761.

SIAP. 2015. (Resumen estatal pecuario). SAGARPA. México. www.siap.gob.mx.

Sierra P., Baca M. Estado del Arte de la Bioeconomía y el Cambio Climático, Cap.3.3 Solar Activity, Agricultural Activity and Climate. Possible links. IBERO-AMERICAN PROGRAMME FOR SCIENCE, TECHNOLOGY AND DEVELOPMENT IBERO-AMERICAN NETWORK OF BIOECONOMICS AND CLMATE CHANGE. Universidad UNAN León, Nicaragua, 2014.

Tenczar P.; Lutz C. C.; Rao V. D.; Goldenfeld N. and Robinson G. E.(2014). Automated monitoring reveals extreme interindividual variation and plasticity in honeybee foraging activity levels. Animal Behaviour 95, (0): 41-48.

vanEngelsdorp D. and Meixner M. D.(2010). A historical review of managed honey bee populations in europe and the united states and the factors that may affect them. Journal of Invertebrate Pathology 103, Supplement, (0): S80-S95. 
Revista Iberoamericana de Bioeconomía y Cambio Climático, 2016, vol. 2, núm. 1, Enero-Julio, ISSN...

Wilson R. and Maclean I. D.(2011). Recent evidence for the climate change threat to lepidoptera and other insects. Journal of Insect Conservation 15, (1-2): 259-268.

Yilmaz M. T.; Tatlisu N. B.; Toker O. S.; Karaman S.; Dertli E.; Sagdic O. and Arici M.(2014). Steady, dynamic and creep rheological analysis as a novel approach to detect honey adulteration by fructose and saccharose syrups: Correlations with hplc-rid results. Food Research International 64: 634-646.

IPCC. (2007). Cambio climático. Bases de la ciencias físicas. In: Solomon S.; Qin D.; Manning M.et als (eds.). Informe del Grupo de Trabajo I. Cambridge, United Kingdom and New York, NY, USA. p. 136. 